\title{
Entanglement entropy in the long-range Kitaev chain
}

\author{
Filiberto Ares* \\ Departamento de Física Teórica, Universidad de Zaragoza, 50009 Zaragoza, Spain \\ José G. Esteve ${ }^{\dagger}$ and Fernando Falceto \\ Departamento de Física Teórica, Universidad de Zaragoza, 50009 Zaragoza, Spain \\ and Instituto de Biocomputación y Física de Sistemas Complejos (BIFI), 50009 Zaragoza, Spain \\ Amilcar R. de Queiroz ${ }^{\S}$ \\ Instituto de Fisica, Universidade de Brasilia, Caixa Postal 04455, 70919-970, Brasília, DF, Brazil
}

(Received 15 February 2018; published 1 June 2018)

\begin{abstract}
In this paper we complete the study on the asymptotic behavior of the entanglement entropy for Kitaev chains with long-range pairing. We discover that when the couplings decay with the distance with a critical exponent new properties for the asymptotic growth of the entropy appear. The coefficient of the leading term is not universal any more and the connection with conformal field theories is lost. We perform a numerical and analytical approach to the problem showing a perfect agreement. In order to carry out the analytical study, a technique for computing the asymptotic behavior of block Toeplitz determinants with discontinuous symbols has been developed.
\end{abstract}

DOI: 10.1103/PhysRevA.97.062301

\section{INTRODUCTION}

Entanglement plays a fundamental role in quantum phase transitions. In one-dimensional critical theories with local interactions, the algebraic decay of the ground-state correlation functions leads to the well-known logarithmic asymptotic growth of the entanglement entropy with a coefficient proportional to the central charge of the underlying conformal field theory [1-3]. On the other hand, outside criticality, the correlations decay exponentially and the entanglement entropy satisfies an area law [4,5].

The presence of long-range interactions radically modifies the previous picture [6,7]. As it is discussed in [8,9] correlations can display exponential and algebraic decay even with nonzero mass gap. This implies that the entanglement entropy may violate the area law while the system is noncritical. This happens in the so-called long-range Kitaev chain [8,9], which has been the object of an intense study in the past years [10-19]. Apart from the theoretical interest, as a model to analyze the role of conformal symmetry in critical points, there has been recently a spectacular development of experimental devices where these long-range interactions can be simulated [20-24].

In [25] we studied the behavior of the entanglement entropy of the vacuum with the size of the subsystem for a long-range Kitaev chain. In order to compute the asymptotic behavior of the entropy we had to establish new results on block Toeplitz determinants with a discontinuous symbol. In that paper we obtained two different regimes depending on the exponent

\footnotetext{
*Corresponding author: ares@unizar.es

†esteve@unizar.es

${ }_{\ddagger}^{\ddagger}$ falceto@unizar.es

§amilcarq@gmail.com
}

$\zeta$ that governs the decay of the couplings. The two regimes were already analyzed numerically in [8] and correspond respectively to $\zeta>1$ and $\zeta<1$. In this paper we study the intermediate critical case $\zeta=1$ for which new physical properties for the entropy show themselves. From the point of view of Toeplitz determinants, the critical exponent poses challenges that must be solved before being able to determine the asymptotic behavior of the entanglement entropy. This task is carried out in the rest of the paper.

\section{MODEL}

The long-range Kitaev chain is a unidimensional homogeneous fermionic chain with nearest-neighbor hopping and powerlike decaying pairings,

$$
\begin{aligned}
H= & \sum_{n=1}^{N}\left(a_{n}^{\dagger} a_{n+1}+a_{n+1}^{\dagger} a_{n}+h a_{n}^{\dagger} a_{n}\right. \\
& \left.+\sum_{|l|<N / 2} \frac{l}{|l|^{\zeta+1}}\left(a_{n}^{\dagger} a_{n+l}^{\dagger}-a_{n} a_{n+l}\right)\right)-\frac{N h}{2},
\end{aligned}
$$

where $a_{n}^{\dagger}, a_{n}$ are fermionic creation and annihilation operators acting on the site $n$, satisfying the canonical anticommutation relations $\left\{a_{n}, a_{m}^{\dagger}\right\}=\delta_{n m},\left\{a_{n}^{\dagger}, a_{m}^{\dagger}\right\}=\left\{a_{n}, a_{m}\right\}=0$ and periodic boundary conditions, that is $a_{n+N}=a_{n}$.

The exponent $\zeta>0$ characterizes the dumping of the coupling with the distance. Its value plays a decisive role in the growth rate of the Rényi entanglement entropy [8,9,25]. In a previous work [25] we analytically studied the leading asymptotic behavior with the size of an interval of the chain $|X|$ when $\zeta \neq 1$. There we found that the entanglement entropy 
$S_{\alpha, X}$ behaves as

$$
S_{\alpha, X}=\frac{\alpha+1}{6 \alpha} c \ln |X|+\cdots
$$

up to finite contributions in the large $|X|$ limit, with an effective central charge $c$ given by

$$
c= \begin{cases}0, & \zeta>1 \text { and } h \neq \pm 2 \\ 1 / 2, & \zeta>1 \text { and } h= \pm 2 \text { or } \zeta<1 \text { and } h \neq 2 \\ 1, & \zeta<1 \text { and } h=2\end{cases}
$$

This is in agreement with the numerical studies previously performed in [8].

We see that the case $\zeta=1$ is the only one not covered in the previous expression. To our knowledge this case has not been considered in the literature in spite of its physical interest as it may be experimentally implemented with chains of magnetic impurities on an $s$-wave superconductor [26]. Our reason to exclude this value in [25] is that in order to address this case we required a technical result that was not available at the moment we wrote the previous paper. In this paper we fill this gap and obtain the scaling behavior in the most general situation. We find that along the line $\zeta=1$ the entanglement entropy grows logarithmically but with a proportionality constant that cannot be expressed in terms of an effective central charge. In the following we will develop the technical tools required for the previous computation.

We must first solve the model in (1). Since it is a translational invariant and quadratic Hamiltonian, one can diagonalize it performing a Fourier plus a Bogoliubov transformation (see, e.g., Ref. [25]). After the latter, the Hamiltonian reads

$$
H=\sum_{k=0}^{N-1} \Lambda_{k} d_{k}^{\dagger} d_{k}
$$

where $d_{k}$ and $d_{k}^{\dagger}$ are also fermionic operators and $\Lambda_{k}$ is the dispersion relation

$$
\Lambda_{k}=\sqrt{\left(h+2 \cos \theta_{k}\right)^{2}+4\left(\sum_{l=1}^{N / 2} \sin \left(l \theta_{k}\right) l^{-\zeta}\right)^{2}}, \quad \theta_{k}=\frac{2 \pi k}{N} .
$$

If we take now the thermodynamic limit $N \rightarrow \infty, \theta_{k}$ is replaced by a continuous variable $\theta \in[-\pi, \pi)$ and the dispersion relation can be expressed as

$$
\Lambda(\theta)=\sqrt{(h+2 \cos \theta)^{2}+\left|G_{\zeta}(\theta)\right|^{2}}
$$

where $G_{\zeta}(\theta) \equiv \Xi_{\zeta}\left(e^{i \theta}\right)$ and

$$
\Xi_{\zeta}(z)=\sum_{l=1}^{\infty}\left(z^{l}-z^{-l}\right) l^{-\zeta}=\operatorname{Li}_{\zeta}(z)-\operatorname{Li}_{\zeta}\left(z^{-1}\right) .
$$

The function $\mathrm{Li}_{\zeta}$ stands for the polylogarithm of order $\zeta$. This is a multivalued function, analytic outside the real interval $[1, \infty)$, and has a finite limit at $z=1$ for $\zeta>1$ while it diverges at that point for $\zeta<1$. For $\zeta=1$ the polylogarithm function reduces to the logarithm $\operatorname{Li}_{1}(z)=-\ln (1-z)$. We shall take as its branch cut the real interval $[1, \infty)$; thus $\Xi_{1}(z)$ has a branch cut along $[0, \infty)$. These properties will be crucial in the study of the entanglement entropy of the ground state of (1).

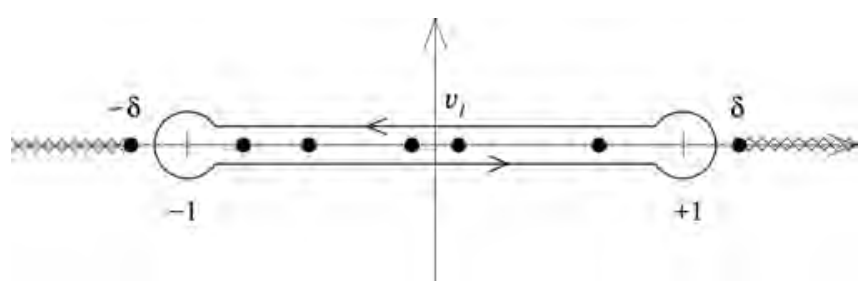

FIG. 1. Contour of integration, cuts, and poles for the computation of $S_{\alpha, X}$. The contour surrounds the eigenvalues $v_{l}$ of $V_{X}$, all of them lying on the real interval $[-1,1]$. The cuts for the function $f_{\alpha}$ extend to $\pm \infty$.

Since $\Lambda_{k} \geqslant 0$, the state of minimum energy $|\mathrm{GS}\rangle$ is the Fock space vacuum for the Bogoliubov modes, i.e., $d_{k}|\mathrm{GS}\rangle=0$ for all $k$. If we split the system in two subchains $X, Y$ of contiguous sites, the Hilbert space factorizes $\mathcal{H}=\mathcal{H}_{X} \otimes \mathcal{H}_{Y}$. Then the Rényi entanglement entropy of $|\mathrm{GS}\rangle$ is defined as

$$
S_{\alpha, X}=\frac{1}{1-\alpha} \ln \operatorname{Tr}\left(\rho_{X}^{\alpha}\right),
$$

with $\rho_{X}=\operatorname{Tr}_{\mathcal{H}_{Y}}|\mathrm{GS}\rangle\langle\mathrm{GS}|$ the reduced density matrix of $X$. As it is well known, for Gaussian theories where the Wick theorem is satisfied, as it is our case, the entanglement entropy of this state can be computed from the correlation matrix [2,27,28]. In fact [29-31],

$$
S_{\alpha, X}=\lim _{\delta \rightarrow 1^{+}} \frac{1}{4 \pi i} \oint_{C} f_{\alpha}(\lambda / \delta) \frac{d}{d \lambda} \ln D_{X}(\lambda) d \lambda,
$$

where

$$
f_{\alpha}(x)=\frac{1}{1-\alpha} \ln \left[\left(\frac{1+x}{2}\right)^{\alpha}+\left(\frac{1-x}{2}\right)^{\alpha}\right],
$$

and $D_{X}(\lambda)=\operatorname{det}\left(\lambda I-V_{X}\right)$ with $V_{X}$ the restriction of the ground-state correlation matrix to the sites that belong to the subsystem $X$,

$$
\left(V_{X}\right)_{n m}=\left\langle\left(\begin{array}{c}
a_{n} \\
a_{n}^{\dagger}
\end{array}\right)\left(a_{m}, a_{m}^{\dagger}\right)\right\rangle-\delta_{n m} I, \quad n, m \in X .
$$

The integration contour and the branch choice for $f_{\alpha}(\lambda / \delta)$ are represented in Fig. 1.

Due to the translational invariance of the Hamiltonian and given the choice of the subsystem $X$, which is composed of contiguous sites, the correlation matrix is a block Toeplitz matrix,

$$
\left(V_{X}\right)_{n m}=\frac{1}{2 \pi} \int_{-\pi}^{\pi} \mathcal{G}(\theta) e^{i \theta(n-m)} d \theta,
$$

generated by a two-dimensional symbol $\mathcal{G}(\theta)$ which, for the ground state of the Hamiltonian (1), has the form

$$
\mathcal{G}(\theta)=\frac{1}{\Lambda(\theta)}\left(\begin{array}{cc}
h+2 \cos \theta & G_{\zeta}(\theta) \\
-G_{\zeta}(\theta) & -h-2 \cos \theta
\end{array}\right) .
$$

By virtue of (5), the asymptotic behavior of $S_{\alpha, X}$ can be obtained from that of the determinant $D_{X}(\lambda)$. The discontinuities of the symbol (8) will give the leading dominant term. In [25], we already computed them for $\zeta \neq 1$, where the lateral limits of each discontinuity commute. For $\zeta=1$ the symbol has a noncommuting discontinuity and we lacked a way to 
determine its contribution. In the following we shall solve this problem.

\section{DETERMINANT OF BLOCK TOEPLITZ MATRICES WITH DISCONTINUOUS SYMBOL}

In this section we shall study the scaling properties of the determinant of a block Toeplitz matrix with a piecewise continuous matrix valued symbol.

Given a $(d \times d)$-dimensional matrix valued function $\mathcal{M}(\theta)$ we shall denote by $T_{X}[\mathcal{M}]$ its associated $(|X| \cdot d)$-dimensional block Toeplitz matrix, i.e., for every $k, l=1, \ldots,|X|$, there is a $d \times d$ block given by

$$
\left(T_{X}[\mathcal{M}]\right)_{k, l}=\frac{1}{2 \pi} \int_{-\pi}^{\pi} \mathcal{M}(\theta) e^{i \theta(k-l)} d \theta .
$$

We shall denote by $D_{X}[\mathcal{M}]=\operatorname{det} T_{X}[\mathcal{M}]$.

From Szegö-Widom theorem [32-34] we know that the leading linear term of $\ln D_{X}[\mathcal{M}]$ is given by

$$
\ln D_{X}[\mathcal{M}]=\frac{|X|}{2 \pi} \int_{-\pi}^{\pi} \ln \operatorname{det} \mathcal{M}(\theta) d \theta+\cdots,
$$

where we assume $\operatorname{det} \mathcal{M}(\theta) \neq 0$ and the dots denote subdominant contributions in the limit $|X| \rightarrow \infty$.

As it is discussed in [25], the next subdominant contribution for a piecewise continuous symbol with jump discontinuities at $\theta_{r}$, for $r=1, \ldots, R$, is logarithmic in $|X|$ and has the form

$$
\begin{aligned}
\ln D_{X}[\mathcal{M}]= & \frac{|X|}{2 \pi} \int_{-\pi}^{\pi} \ln \operatorname{det} \mathcal{M}(\theta) d \theta \\
& +\ln |X| \sum_{r=1}^{R} b_{r}+\cdots,
\end{aligned}
$$

where the coefficient $b_{r}$ depends uniquely on the lateral limits at the discontinuity $\theta_{r}$.

In the particular case in which the two lateral limits commute an explicit expression for $b_{r}$ is proposed in [25]. We review here this result.

If for a discontinuity $\theta_{r}$ the two lateral limits

$$
\mathcal{M}_{r}^{-}=\lim _{\theta \rightarrow \theta_{r}^{-}} \mathcal{M}(\theta), \quad \mathcal{M}_{r}^{+}=\lim _{\theta \rightarrow \theta_{r}^{+}} \mathcal{M}(\theta)
$$

commute and are diagonalizable, then we can diagonalize both in the same basis. Let us denote by $\mu_{r, j}^{ \pm}, j=1, \ldots, d$ the corresponding eigenvalues for each lateral limit of the discontinuity at $\theta_{r}$. Then inspired by the similar case for a scalar symbol [35-37], we conjectured in [25] that the logarithmic coefficients are given by

$$
b_{r}=\frac{1}{4 \pi^{2}} \sum_{j=1}^{d}\left(\ln \frac{\mu_{r, j}^{-}}{\mu_{r, j}^{+}}\right)^{2} .
$$

This conjecture was verified numerically to all attainable precision. Note that the previous expression can be written in the more compact (and meaningful) form

$$
b_{r}=\frac{1}{4 \pi^{2}} \operatorname{Tr}\left[\ln \mathcal{M}_{r}^{-}\left(\mathcal{M}_{r}^{+}\right)^{-1}\right]^{2} .
$$

In the following we shall argue that if this result is true, it is also valid when the two lateral limits do not commute.
In order to show it, we have to invoke an old result by Widom [33] that, in the particular case that concerns our derivation, can be stated in the following very simple form: for any $d \times d$ constant matrix $\mathcal{C}$ and any symbol $\mathcal{M}(\theta)$ as before we have

$$
T_{X}[\mathcal{M C}]=T_{X}[\mathcal{M}] T_{X}[\mathcal{C}]
$$

Actually $T_{X}[\mathcal{C}]=I_{|X|} \otimes \mathcal{C}$ and the relation above immediately follows. For the determinants we have

$$
\ln D_{X}[\mathcal{M C}]=\ln D_{X}[\mathcal{M}]+|X| \ln \operatorname{det} \mathcal{C} .
$$

Now, in the general case of a piecewise continuous symbol with noncommuting lateral limits, $\mathcal{M}_{r}^{ \pm}$, at the discontinuity point $\theta_{r}$, we can apply the previous result choosing the constant symbol $\mathcal{C}=\left(\mathcal{M}_{r}^{+}\right)^{-1}$. Hence we have

$$
\ln D_{X}[\mathcal{M}]=\ln D_{X}\left[\mathcal{M}\left(\mathcal{M}_{r}^{+}\right)^{-1}\right]+|X| \ln \operatorname{det} \mathcal{M}_{r}^{+} .
$$

Now, applying the expansion in (9) to the new symbol $\mathcal{M}\left(\mathcal{M}_{r}^{+}\right)^{-1}$ we have

$$
\begin{aligned}
\ln D_{X}\left[\mathcal{M}\left(\mathcal{M}_{r}^{+}\right)^{-1}\right]= & \frac{|X|}{2 \pi} \int_{-\pi}^{\pi} \ln \operatorname{det} \mathcal{M}(\theta) d \theta \\
& -|X| \ln \operatorname{det} \mathcal{M}_{r}^{+} \\
& +\ln |X| \sum_{r=1}^{R} b_{r}^{\prime}+\cdots .
\end{aligned}
$$

But the two lateral limits at $\theta_{r}$ are $\mathcal{M}_{r}^{-}\left(\mathcal{M}_{r}^{+}\right)^{-1}$ and $I$ which, of course, commute. Therefore, we can apply the previous result on commuting lateral symbols and we obtain

$$
b_{r}^{\prime}=\frac{1}{4 \pi^{2}} \operatorname{Tr}\left[\ln \mathcal{M}_{r}^{-}\left(\mathcal{M}_{r}^{+}\right)^{-1}\right]^{2} .
$$

If we combine (9), (12), and (13), we get $b_{r}=b_{r}^{\prime}$ and therefore (11) is valid even if the lateral limits do not commute, as stated.

\section{ENTANGLEMENT ENTROPY}

After the technical parenthesis of the previous section we continue with the main goal of the paper. Our task is to obtain the scaling behavior of the entanglement entropy $S_{\alpha, X}$ as derived from formula (5).

For that purpose we must use the results of the previous section to compute the block Toeplitz determinant $D_{X}(\lambda)$ or, equivalently, $D_{X}\left[\mathcal{G}_{\lambda}\right]$ in the notation of the last section. Here we have introduced the two-dimensional symbol $\mathcal{G}_{\lambda}(\theta)=\lambda I-$ $\mathcal{G}(\theta)$ with $\mathcal{G}(\theta)$ taken from $(8)$.

The linear term of $\ln D_{X}(\lambda)$ is derived from the Szegö estimate and, given that $\operatorname{det} \mathcal{G}_{\lambda}(\theta)=\lambda^{2}-1$, the asymptotic behavior is

$$
\ln D_{X}(\lambda)=|X| \ln \left(\lambda^{2}-1\right)+\ln |X| \sum_{r} b_{r}(\lambda)+\cdots,
$$

where as before the dots represent finite terms in the large $|X|$ limit and the coefficients of the logarithmic piece are associated to the discontinuities of $\mathcal{G}_{\lambda}$ by the expression derived in the previous section. Namely, the coefficient $b_{r}(\lambda)$ associated to the discontinuity at $\theta_{r}$ is given by

$$
b_{r}(\lambda)=\frac{1}{4 \pi^{2}} \operatorname{Tr}\left[\ln \mathcal{G}_{\lambda, r}^{-}\left(\mathcal{G}_{\lambda, r}^{+}\right)^{-1}\right]^{2} .
$$


Therefore, to proceed we must identify the location of the jumps of $\mathcal{G}_{\lambda}$ and compute the lateral limits.

It is clear that the possible sources of discontinuities for $\mathcal{G}_{\lambda}$ are the discontinuities or the zeros of $\Lambda(\theta)$. In our case of interest, $\zeta=1$, these are $\theta=0$, for which

$$
G_{\zeta=1}(\theta)=i(\pi-\theta), \quad \theta \in[0,2 \pi)
$$

has a jump and, for $h=2, \theta=\pi$, where $\Lambda(\theta)$ vanishes. We will discuss separately both discontinuity points.

Let us call $b_{0}$ the coefficient in the expansion of $\ln D_{X}(\lambda)$ associated to $\theta=0$. The two lateral limits are conveniently expressed in terms of the Pauli sigma matrices

$$
\mathcal{G}_{\lambda, 0}^{ \pm}=\lambda I-\cos \xi \sigma_{z} \pm \sin \xi \sigma_{y},
$$

where

$$
\cos \xi=\frac{h+2}{\sqrt{(h+2)^{2}+\pi^{2}}}, \quad \sin \xi=\frac{\pi}{\sqrt{(h+2)^{2}+\pi^{2}}} .
$$

Clearly for $\xi \neq \pi / 2$ the two limits do not commute and we are bound to use the results of the previous section.

With a little algebra we get

$$
\begin{aligned}
& \mathcal{G}_{\lambda, 0}^{-}\left(\mathcal{G}_{\lambda, 0}^{+}\right)^{-1} \\
& \quad=\frac{1}{\lambda^{2}-1}\left[\left(\lambda^{2}-\cos 2 \xi\right) I-2 \lambda \sin \xi \sigma_{y}-i \sin \xi \sigma_{x}\right] .
\end{aligned}
$$

In order to compute $b_{0}(\lambda)$ we need the eigenvalues $\mu_{ \pm}(\lambda)$ of the previous matrix. They can be written

$$
\mu_{ \pm}(\lambda)=\left(\frac{\sqrt{\lambda^{2}-\cos ^{2} \xi} \pm \sin \xi}{\sqrt{\lambda^{2}-1}}\right)^{2}
$$

Notice also that we have

$$
\mu_{+}(\lambda)=\mu_{-}(\lambda)^{-1}
$$

Putting everything together we finally obtain

$$
\begin{aligned}
b_{0}(\lambda) & =\frac{1}{4 \pi^{2}}\left[\left(\ln \mu_{+}(\lambda)\right)^{2}+\left(\ln \mu_{-}(\lambda)\right)^{2}\right] \\
& =\frac{2}{\pi^{2}}\left(\ln \frac{\sqrt{\lambda^{2}-\cos ^{2} \xi}+\sin \xi}{\sqrt{\lambda^{2}-1}}\right)^{2} .
\end{aligned}
$$

As we discussed before, for $h \neq 2$ this is the only discontinuity of the symbol and therefore the only contribution to the logarithmic term for $\ln D_{X}(\lambda)$. Namely,

$$
\ln D_{X}(\lambda)=|X| \ln \left(\lambda^{2}-1\right)+\ln |X| b_{0}(\lambda)+\cdots .
$$

This must be inserted into (5) to derive the scaling behavior of the entanglement entropy.

The linear term is obtained from the integral

$$
\lim _{\delta \rightarrow 1^{+}} \frac{1}{2 \pi \mathrm{i}} \oint_{C} f_{\alpha}(\lambda / \delta) \frac{\lambda}{\lambda^{2}-1} d \lambda=\frac{1}{2}\left[f_{\alpha}(1)+f_{\alpha}(-1)\right]=0,
$$

where Cauchy's residue theorem has been used and from the expression in (6) we immediately see that $f_{\alpha}( \pm 1)=0$.

The previous result: vanishing of the term that scales linearly with the size of the subsystem is in agreement with the area law that states that in the ground state of a local theory the entanglement entropy is not an extensive quantity. As we will see now the area law has corrections and the entanglement

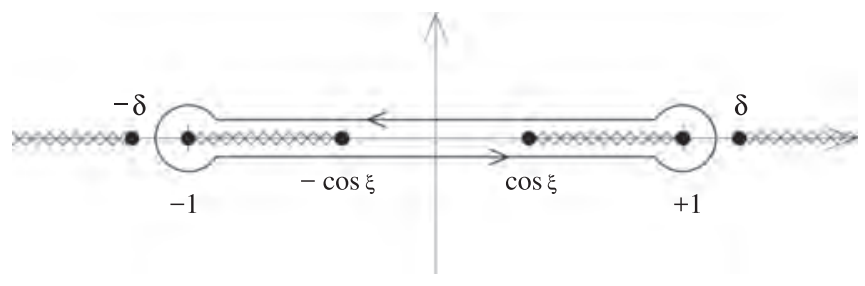

FIG. 2. Contour of integration and cuts of the integrand in (15) for the computation of $B_{\alpha, 0}$. The cuts from $\pm \delta$ to the infinity correspond to $d f_{\alpha}(\lambda / \delta) / d \lambda$, while the cuts inside the contour, $[-1,-\cos \xi]$ and [cos $\xi, 1]$, are due to the other factor of the integrand.

entropy scales with the logarithm of the size of the subsystem rather than going to a constant.

The coefficient of the logarithmic term for the entropy, that we denote by $B_{\alpha}$, gets just one contribution, named $B_{\alpha, 0}$, from the discontinuity at $\theta=0$; we are considering $h \neq 2$. It can be computed from (5)

$$
\begin{aligned}
B_{\alpha, 0}= & \lim _{\delta \rightarrow 1^{+}} \frac{1}{4 \pi i} \oint_{C} f_{\alpha}(\lambda / \delta) \frac{d b_{0}(\lambda)}{d \lambda} d \lambda \\
= & -\lim _{\delta \rightarrow 1^{+}} \frac{1}{2 \pi^{3} i} \oint_{C} \frac{d f_{\alpha}(\lambda / \delta)}{d \lambda} \\
& \times\left(\ln \frac{\sqrt{\lambda^{2}-\cos ^{2} \xi}+\sin \xi}{\sqrt{\lambda^{2}-1}}\right)^{2} d \lambda,
\end{aligned}
$$

where, for convenience, we have performed an integration by parts. The branch cuts of the different multivalued functions involved in the integral are depicted in Fig. 2.

Now it is possible to take the limit in (15) and, given that $f_{\alpha}$ is an even function, the complex integral can be reduced to the following real one:

$$
B_{\alpha, 0}=\frac{2}{\pi^{2}} \int_{\cos \xi}^{1} \frac{d f_{\alpha}(\lambda)}{d \lambda} \ln \frac{\sqrt{1-\lambda^{2}}}{\sqrt{\lambda^{2}-\cos ^{2} \xi}+\sin \xi} d \lambda,
$$

where we take positive square roots. Note that for integer $\alpha>1$

$$
\frac{d f_{\alpha}(\lambda)}{d \lambda}=\frac{\alpha}{1-\alpha} \frac{(1+\lambda)^{\alpha-1}-(1-\lambda)^{\alpha-1}}{(1+\lambda)^{\alpha}+(1-\lambda)^{\alpha}}
$$

is a meromorphic function with poles along the imaginary axis located at

$$
\lambda_{k}=i \tan \frac{\pi(2 k-1)}{2 \alpha}, \quad k=1,2, \ldots, \alpha, \quad \text { with } k \neq \frac{\alpha+1}{2} .
$$

Hence, by sending the integration contour in (15) to infinity, we can reduce the integral to the computation of the corresponding residues. In this way we get a completely explicit expression for $B_{\alpha, 0}$ (valid only for integer $\alpha>1$ )

$$
B_{\alpha, 0}=\frac{1}{\pi^{2}(\alpha-1)} \sum_{k=1}^{\alpha} \arctan ^{2} \frac{\sin \xi}{\sqrt{\cos ^{2} \xi+\left|\lambda_{k}\right|^{2}}} .
$$

In particular, for $\alpha=2,3$, the sum in the previous expression reduces to

$$
\begin{aligned}
& B_{2,0}=\frac{2}{\pi^{2}} \arctan ^{2} \frac{\sin \xi}{\sqrt{\cos ^{2} \xi+1}}, \\
& B_{3,0}=\frac{1}{\pi^{2}} \arctan ^{2} \frac{\sin \xi}{\sqrt{\cos ^{2} \xi+1 / 3}} .
\end{aligned}
$$




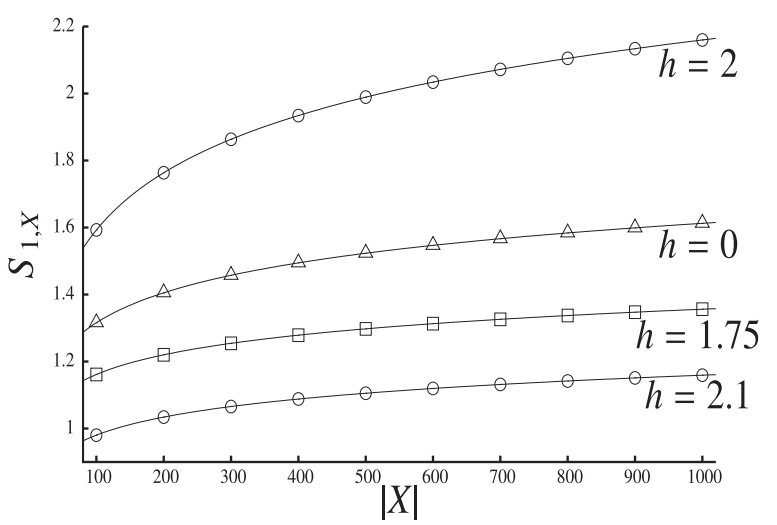

FIG. 3. Numerical check of the asymptotic scaling of the von Neumann entanglement entropy $(\alpha=1)$ with the length of the interval $|X|$ for $\zeta=1$ and different values of $h$. The dots represent the numerical computation, while the solid lines correspond to the curve $B_{1} \ln |X|+$ const, where $B_{1}=B_{1,0}$ for $h \neq 2$ and $B_{1}=B_{1,0}+1 / 6$ if $h=2$. $B_{1,0}$ is given by (16). The constant is obtained by fitting the numerical and the analytical results at $|X|=1000$.

In Fig. 3 we numerically check the scaling of the entanglement entropy given by the expression (16) that we have just obtained for the logarithmic coefficient $B_{\alpha}$. Although we have only plotted the von Neumann entropy, we have seen that the agreement with the numerical computations is just as good for other values of $\alpha$.

For local theories it is possible to relate the entanglement entropy to universal properties of conformal field theories. In this case the coefficient of the logarithmic term in the expansion should read

$$
B_{\alpha}=\frac{\alpha+1}{6 \alpha} c,
$$

where $c$, the central charge, is a constant that depends on the universality class of the underlying conformal field theory. In particular, this relation implies

$$
B_{2}=\frac{9}{8} B_{3},
$$

something that, for general $h$, does not hold in our case. The reason for that could be that couplings in our theory have infinite range and therefore it cannot be related to any local field theory. However, as it was shown in [25], when the coupling constants decay faster $(\zeta>1)$ or slower $(\zeta<1)$ than our critical case, relation (18) also holds, even if the theory is not local. Another instance in which (18) holds is when $h=-2$; then $\xi=\pi / 2$ and we have

$$
B_{2,0}=\frac{1}{8}, \quad B_{3,0}=\frac{1}{9},
$$

which fulfill (18) with $c=1 / 2$.

This is the whole story about the logarithmic coefficient, and $B_{\alpha}=B_{\alpha, 0}$ for $h \neq 2$, as we discussed before. However, for $h=2$, the dispersion relation $\Lambda(\theta)$ vanishes at $\theta=\pi$ and it induces a discontinuity in the symbol. The two lateral limits are

$$
\mathcal{G}_{\lambda, \pi}^{ \pm}=\lambda I \pm \sigma_{y}
$$

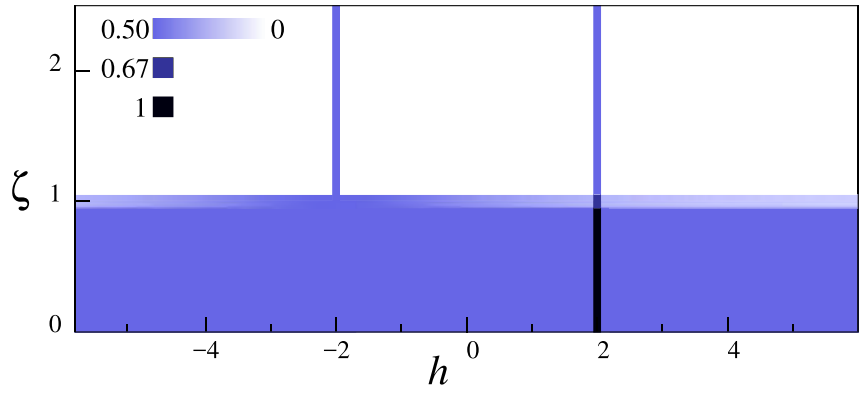

FIG. 4. Plot of the different regions in the $(h, \zeta)$ plane according to the coefficient $B_{2}$ of the logarithmic scaling for the entanglement entropy. The coloring stands for the value of $2 B_{2}$ : from white for $B_{2}=0$ to black when $B_{2}=1 / 2$.

and its contribution to the logarithmic term in the expansion of $\ln D_{X}(\lambda)$ is given by

$$
\begin{aligned}
b_{\pi}(\lambda) & =\frac{1}{4 \pi^{2}} \operatorname{Tr}\left[\ln \mathcal{G}_{\lambda, \pi}^{-}\left(\mathcal{G}_{\lambda, \pi}^{+}\right)^{-1}\right]^{2} \\
& =\frac{1}{2 \pi^{2}}\left(\ln \frac{\lambda+1}{\lambda-1}\right)^{2}
\end{aligned}
$$

Associated to that there is a new logarithmic term for the entropy whose coefficient is given by

$$
B_{\alpha, \pi}=\lim _{\delta \rightarrow 1^{+}} \frac{1}{4 \pi i} \oint_{C} f_{\alpha}(\lambda / \delta) \frac{d}{d \lambda} b_{\pi}(\lambda) d \lambda=\frac{\alpha+1}{12 \alpha} .
$$

In the light of the previous discussion this contribution to the logarithmic term can be interpreted as coming from a conformal field theory with central charge $c=1 / 2$.

We would like to stress again that the last interpretation is valid only for part of the contribution to the logarithmic term of the entropy. One should consider the whole coefficient $B_{\alpha}=$ $B_{\alpha, 0}+B_{\alpha, \pi}$, that includes the discontinuity at $\theta=0$, and this, for $h=2$, does not behave as predicted by the conformal field theory under variations of $\alpha$. Observe that in Fig. 3 we have considered this particular point. Also in this case the analytical formulas we have obtained are in agreement with the numerical results.

As a summary, in Fig. 4 we have recollected the previous known results (2) and (3) outside the line $\zeta=1$ together with those obtained in this section for $\zeta=1$. We have colored the parameter space $(h, \zeta)$ according to the value of the logarithmic term $B_{\alpha}$. The model is critical, and the mass gap is zero, only in the lines $h= \pm 2$. Note, however, that the entanglement entropy scales logarithmically for $\zeta \leqslant 1$ even outside criticality.

As a further check of the results obtained in the previous section we shall consider a variant of the long-range Kitaev chain. Namely we shall discuss the case in which we have two singularities in the symbol (instead of one) located at $\theta= \pm \phi$. One reason for this is connected to the study of the Möbius symmetry introduced in [38,39]. While $\theta=0$ is a fixed point of the above-mentioned transformations $\theta= \pm \phi$ is not, which enriches the symmetry. We do not pursue the analysis of Möbius transformations in this paper. 
The new singularities in the symbol can be obtained by adding to (1) an oscillatory factor in the pairing,

$$
\begin{aligned}
H^{\prime}= & \sum_{n=1}^{N}\left(a_{n}^{\dagger} a_{n+1}+a_{n+1}^{\dagger} a_{n}+h a_{n}^{\dagger} a_{n}\right. \\
& \left.+\sum_{|l|<N / 2} \frac{l \cos (l \phi)}{|l|^{\zeta+1}}\left(a_{n}^{\dagger} a_{n+l}^{\dagger}-a_{n} a_{n+l}\right)\right)-\frac{N h}{2},
\end{aligned}
$$

with $\phi \in[0, \pi)$. is

In the thermodynamic limit $N \rightarrow \infty$, the dispersion relation

$$
\Lambda^{\prime}(\theta)=\sqrt{(h+2 \cos \theta)^{2}+\left|G_{\zeta, \phi}(\theta)\right|^{2}},
$$

where $G_{\zeta, \phi}(\theta)=\Xi_{\zeta, \phi}\left(e^{i \theta}\right)$, and

$$
\begin{aligned}
G_{\zeta, \phi}(\theta)= & \sum_{l=1}^{\infty} \frac{\cos (l \phi)}{l^{\zeta}}\left(e^{i \theta l}-e^{-i \theta l}\right) \\
= & \frac{1}{2}\left[\operatorname{Li}_{\zeta}\left(e^{i(\phi+\theta)}\right)-\operatorname{Li}_{\zeta}\left(e^{i(\phi-\theta)}\right)\right. \\
& \left.+\operatorname{Li}_{\zeta}\left(e^{-i(\phi-\theta)}\right)-\operatorname{Li}_{\zeta}\left(e^{-i(\phi+\theta)}\right)\right] .
\end{aligned}
$$

This function vanishes at $\theta=0$ and $\pi$, it is smooth for $\zeta>1$, diverges at $\theta= \pm \phi$ for $\zeta<1$, and for our particular case of interest in this paper, $\zeta=1$, it reads

$$
G_{1, \phi}(\theta)= \begin{cases}-i(\pi+\theta), & -\pi \leqslant \theta \leqslant-\phi, \\ -i \theta, & -\phi<\theta<\phi, \\ i(\pi-\theta), & \phi \leqslant \theta<\pi .\end{cases}
$$

It has a jump at $\theta=\phi$ with lateral limits $i \phi$ and $i(\pi-\phi)$ and another one at $\theta=-\phi$ with limits $i(\phi-\pi)$ and $-i \phi$.

The symbol of the correlation matrix can be written

$$
\mathcal{G}^{\prime}(\theta)=\frac{1}{\Lambda^{\prime}(\theta)}\left(\begin{array}{cc}
h+2 \cos \theta & G_{\zeta, \phi}(\theta) \\
-G_{\zeta, \phi}(\theta) & -h-2 \cos \theta
\end{array}\right) .
$$

For $h \neq \pm 2$ and $\zeta=1$ the only jumps of $\mathcal{G}_{\lambda}^{\prime}(\theta)=\lambda I-\mathcal{G}^{\prime}(\theta)$ take place at $\theta= \pm \phi$. We shall compute separately the contribution of each discontinuity to the coefficient of the logarithmic term in the expansion of the entanglement entropy.

In the first place we consider the point $\theta=\phi$. The lateral limits of $\mathcal{G}_{\lambda}^{\prime}(\theta)$ are

$$
\mathcal{G}_{\lambda, \phi}^{\prime \pm}=\lambda I-\cos \xi^{ \pm} \sigma_{z}-\sin \xi^{ \pm} \sigma_{y}
$$

where

$$
\begin{aligned}
\cos \xi^{+} & =\frac{h+2 \cos \phi}{\sqrt{(h+2 \cos \phi)^{2}+(\phi-\pi)^{2}}}, \\
\sin \xi^{+} & =\frac{\phi-\pi}{\sqrt{(h+2 \cos \phi)^{2}+(\phi-\pi)^{2}}}
\end{aligned}
$$

and

$$
\begin{aligned}
& \cos \xi^{-}=\frac{h+2 \cos \phi}{\sqrt{(h+2 \cos \phi)^{2}+\phi^{2}}}, \\
& \sin \xi^{-}=\frac{\phi}{\sqrt{(h+2 \cos \phi)^{2}+\phi^{2}}} .
\end{aligned}
$$

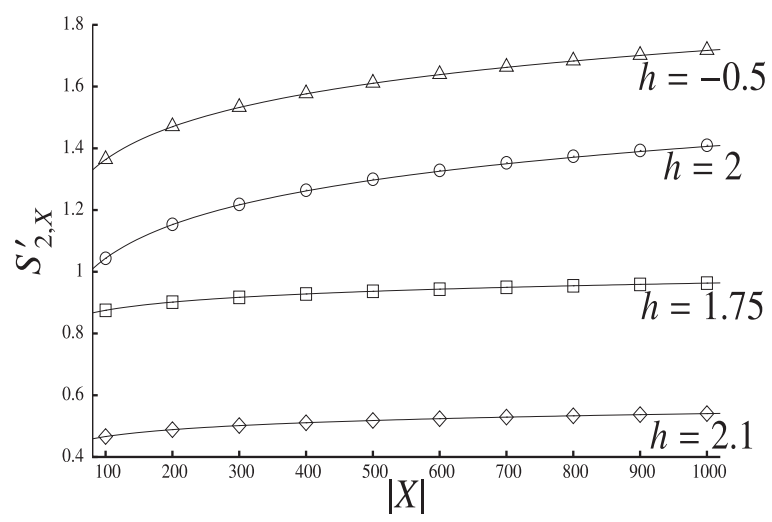

FIG. 5. Rényi entanglement entropy with $\alpha=2$ as a function of the length $|X|$ of the interval for $\phi=\pi / 4, \zeta=1$, and different values of $h$. The dots correspond to the numerical entropy, while the solid lines are our analytical prediction $B_{2}^{\prime} \ln |X|+$ const with $B_{2}^{\prime}$ that is obtained in the text: $B_{2}^{\prime}=2 B_{2, \pi / 4}^{\prime}+1 / 4$ for $h= \pm 2$ and $B_{2}^{\prime}=2 B_{2, \pi / 4}^{\prime}$ with $B_{2, \pi / 4}$ given by (23). The constant is obtained by fitting the numerical and the analytical results at $|X|=1000$.

The eigenvalues of $\mathcal{G}_{\lambda, \phi}^{\prime-}\left(\mathcal{G}_{\lambda, \phi}^{\prime+}\right)^{-1}$ are

$$
\mu_{ \pm}^{\prime}(\lambda)=\left(\frac{\sqrt{\lambda^{2}-\cos ^{2}(\Delta \xi / 2)} \pm \sin (\Delta \xi / 2)}{\sqrt{\lambda^{2}-1}}\right)^{2},
$$

where $\Delta \xi=\xi^{+}-\xi^{-}$. Observe that the expression for the eigenvalues is exactly that of the previous model, with the only change of $\xi$ by $\Delta \xi / 2$.

Therefore, the contribution of this discontinuity to the logarithmic coefficient of the entanglement entropy, $B_{\alpha, \phi}^{\prime}$, can be written

$$
\begin{aligned}
B_{\alpha, \phi}^{\prime}= & \frac{2}{\pi^{2}} \int_{\cos \frac{\Delta \xi}{2}}^{1} \frac{d f_{\alpha}(\lambda)}{d \lambda} \\
& \times \ln \frac{\sqrt{1-\lambda^{2}}}{\sqrt{\lambda^{2}-\cos ^{2}(\Delta \xi / 2)}+\sin (\Delta \xi / 2)} d \lambda,
\end{aligned}
$$

and the corresponding integrated expression for integer $\alpha>1$

$$
B_{\alpha, \phi}^{\prime}=\frac{1}{\pi^{2}(\alpha-1)} \sum_{k=1}^{\alpha} \arctan ^{2} \frac{\sin (\Delta \xi / 2)}{\sqrt{\cos ^{2}(\Delta \xi / 2)+\left|\lambda_{k}\right|^{2}}} .
$$

The other discontinuity point, $\theta=-\phi$, can be computed along the same lines. Actually the only difference with respect to the previous case is that we have to replace $\xi^{+}$and $\xi^{-}$with $-\xi^{-}$ and $-\xi^{+}$, respectively. Hence $\Delta \xi$ is unchanged, $B_{\alpha,-\phi}^{\prime}=B_{\alpha, \phi}^{\prime}$, and for $h \neq \pm 2$ the asymptotic behavior of the entanglement entropy for this modified long-range Kitaev chain is

$$
S_{\alpha, X}^{\prime}=2 B_{\alpha, \phi}^{\prime} \ln |X|+\cdots,
$$

with $B_{\alpha, \phi}^{\prime}$ given above. In Fig. 5 we compare the numerical value of the entanglement entropy for different values of $h$ along the line $\zeta=1$ when $\alpha=2$ and $\phi=\pi / 4$. The agreement between them makes us conclude that the expression that we have obtained for the leading contribution of the discontinuities of a block Toeplitz matrix is correct.

For completeness we also consider the case $h= \pm 2$ that was excluded in the previous considerations. The Hamiltonian 
in this case is gapless and the coefficient in the expansion of the entanglement entropy gets an extra coefficient due to this fact. In both cases $h=2$ or -2 the extra term is the same and coincides with the one we obtained previously. Namely, for $h= \pm 2$ we have

$$
B_{\alpha}^{\prime}=2 B_{\alpha, \phi}^{\prime}+\frac{\alpha+1}{12 \alpha} .
$$

In Fig. 5 we also checked the case $h=2$ when $\alpha=2$. Observe that the numerical values agree with the form of $B_{\alpha}^{\prime}$ deduced for this case.

\section{CONCLUSIONS}

In this work, we have completed the study of the scaling behavior of the ground-state entanglement entropy in the long-range Kitaev chain that we started in [25]. Our analysis is based on the relation between the entanglement entropy and the determinant of the correlation matrix. Since the chain is translational invariant, the correlation matrix for a single interval is block Toeplitz.

If the symbol of the block Toeplitz matrix is continuous, the entropy scales with the area of the subsystem and hence has a finite asymptotic limit. On the contrary, the presence of discontinuities gives rise to corrections to the area law, leading to a logarithmic growth of the entropy with the size of the interval. In [25] we considered the case $\zeta \neq 1$. There, we were able to compute the asymptotic behavior of the entanglement entropy thanks to the fact that the lateral limits of the jump discontinuities commute in that case. However, for the intermediate value $\zeta=1$ we have jump discontinuities whose lateral limits do not commute and the results of [25] cannot be applied. Motivated by this physical problem, we have investigated more deeply into the theory of block Toeplitz determinants with discontinuous symbols and finally we have produced a general expression for the leading contribution to the determinant of the discontinuities (both commutative and noncommutative) in the matrix symbol. We have checked this result numerically and we find a complete agreement with the theoretical predictions.

A striking feature of the entanglement entropy in the intermediate case is that it cannot be derived from a conformal field theory. Actually, in the noncritical case, $\zeta \neq 1$, the coefficient of the leading logarithmic term of the Rényi entanglement entropy can be derived from a conformal field theory thanks to the replica trick. This fixes the dependence of the coefficient on the Rényi exponent and the only free parameter corresponds to the effective central charge of the underlying conformal theory. On the contrary, in the intermediate case, $\zeta=1$, the logarithmic terms originated in noncommutative discontinuities show a different dependence on the Rényi exponent and therefore cannot be related to a conformal field theory.

It would be nice to understand better this property. A starting point could be the analysis performed in $[8,9]$ on the asymptotic form of the correlations for fermionic chains with long-range pairing.

\section{ACKNOWLEDGMENTS}

Research partially supported by Grants No. 2016-E24/2, No. DGIID-DGA, and No. FPA2015-65745-P, MINECO (Spain). F.A. is supported by FPI Fellowship No. C070/2014, DGIID-DGA/European Social Fund.
[1] C. Holzhey, F. Larsen, and F. Wilczek, Geometric and renormalized entropy in conformal field theory, Nucl. Phys. B 424, 443 (1994).

[2] G. Vidal, J. I. Latorre, E. Rico, and A. Kitaev, Entanglement in Quantum Critical Phenomena, Phys. Rev. Lett. 90, 227902 (2003).

[3] P. Calabrese and J. Cardy, Entanglement entropy and quantum field theory, J. Stat. Mech. (2004) P06002.

[4] M. B. Hastings, An area law for one-dimensional quantum systems, J. Stat. Mech. (2007) P08024.

[5] F. G. S. L. Brandao and M. Horodecki, An area law for entanglement from exponential decay of correlations, Nat. Phys. 9, 721 (2013).

[6] X.-L. Deng, D. Porras, and J. I. Cirac, Effective spin quantum phases in systems of trapped ions, Phys. Rev. A 72, 063407 (2005).

[7] T. Koffel, M. Lewenstein, and L. Tagliacozzo, Entanglement Entropy for the Long-Range Ising Chain in a Transverse Field, Phys. Rev. Lett. 109, 267203 (2012).

[8] D. Vodola, L. Lepori, E. Ercolessi, A. V. Gorshkov, and G. Pupillo, Kitaev Chains with Long-Range Pairing, Phys. Rev. Lett. 113, 156402 (2014).

[9] D. Vodola, L. Lepori, E. Ercolessi, and G. Pupillo, Long-range Ising and Kitaev models: Phases, correlations and edge modes, New J. Phys. 18, 015001 (2016).
[10] L. Lepori, D. Vodola, G. Pupilo, G. Gori, and A. Trombettoni, Effective theory and breakdown of conformal symmetry in a long-range quantum chain, Ann. Phys. (NY) 374, 35 (2016).

[11] M. Van Regemortel, D. Sels, and M. Wouters, Information propagation and equilibration in long-range Kitaev chains, Phys. Rev. A 93, 032311 (2016).

[12] L. Lepori, A. Trombettoni, and D. Vodola, Singular dynamics and emergence of nonlocality in long-range quantum models, J. Stat. Mech. (2017) 033102.

[13] K. Patrick, T. Neupert, and J. K. Pachos, Topological Quantum Liquids with Long-Range Couplings, Phys. Rev. Lett. 118, 267002 (2017).

[14] L. Lepori and L. Dell' Anna, Long-range topological insulators and weakened bulk-boundary correspondence, New J. Phys. 19, 103030 (2017).

[15] S. Hernández-Santana, C. Gogolin, J. I. Cirac, and A. Acín, Correlation Decay in Fermionic Lattice Systems with PowerLaw Interactions at Non-Zero Temperature, Phys. Rev. Lett. 119, 110601 (2017).

[16] A. Alecce and L. Dell'Anna, Extended Kitaev chain with longerrange hopping and pairing, Phys. Rev. B 95, 195160 (2017).

[17] N. Sedlmayr, P. Jäger, M. Maiti, and J. Sirker, A bulkboundary correspondence for dynamical phase transitions in one-dimensional topological insulators and superconductors, Phys. Rev. B 97, 064304 (2018). 
[18] O. Viyuela, D. Vodola, G. Pupillo, and M. A. Martín-Delgado, Topological massive dirac edge modes and long-range superconducting hamiltonians, Phys. Rev. B 94, 125121 (2016).

[19] P. Cats, A. Quelle, O. Viyuela, M. A. Martín-Delgado, and C. Morais-Smith, Staircase to higher topological phase transitions, Phys. Rev. B 97, 121106(R) (2018).

[20] A. Micheli, G. K. Brennen, and P. Zoller, A toolbox for lattice spin models with polar molecules, Nat. Phys. 2, 341 (2006).

[21] R. Islam, E. E. Edwards, K. Kim, S. Korenblit, C. Noh, H. Carmichael, G.-D. Lin, L.-M. Duan, C.-C. Joseph Wang, J. K. Freericks, and C. Monroe, Onset of a quantum phase transition with a trapped ion quantum simulator, Nat. Commun. 2, 377 (2011).

[22] J. W. Britton, B. C. Sawyer, A. C. Keith, C.-C. J. Wang, J. K. Freericks, H. Uys, M. J. Biercuk, and J. J. Bollinger, Engineered two-dimensional Ising interactions in a trapped-ion quantum simulator with hundreds of spins, Nature (London) 484, 489 (2012).

[23] P. Richerme, Z.-X. Gong, A. Lee, C. Senko, J. Smith, M. FossFeig, S. Michalakis, A. V. Gorshkov, and C. Monroe, Non-local propagation of correlations in long-range interacting quantum systems, Nature (London) 511, 198 (2014).

[24] H. Labuhn, D. Barredo, S. Ravets, S. de Léséleuc, T. Macrì, T. Lahaye, and A. Browaeys, Realizing quantum Ising models in tunable two-dimensional arrays of single Rydberg atoms, Nature (London) 534, 667 (2016).

[25] F. Ares, J. G. Esteve, F. Falceto, and A. R. de Queiroz, Entanglement in fermionic chains with finite range coupling and broken symmetries, Phys. Rev. A 92, 042334 (2015).

[26] F. Pientka, L. I. Glazman, and F. von Oppen, Topological superconducting phase in helical Shiba chains, Phys. Rev. B 88, 155420 (2013).

[27] R. D. Sorkin, On the entropy of the vacuum outside a horizon, $X$ Conference on General Relativity and Gravitation, Contributed
Papers (unpublished), Vol. II, pp. 734-736, arXiv:1402.3589 [gr-qc].

[28] I. Peschel, Calculation of reduced density matrices from correlation functions, J. Phys. A: Math. Gen. 36, L205 (2003).

[29] B.-Q. Jin and V. E. Korepin, Quantum spin chain, toeplitz determinants and fisher-hartwig conjecture, J. Stat. Phys. 116, 79 (2004).

[30] A. R. Its, B. Q. Jin, and V. E. Korepin, Entropy of XY spin chain and block toeplitz determinants, Fields Institute Communications, Universality and Renormalization, Vol. 50 (American Mathematical Society, Providence, Rhode Island, 2007), p. 151.

[31] A. R. Its, F. Mezzadri, and M. Y. Mo, Entanglement entropy in quantum spin chains with finite range interaction, Commun. Math. Phys. 284, 117 (2008).

[32] H. Widom, Asymptotic behavior of block Toeplitz matrices and determinants, Adv. Math. 13, 284 (1974).

[33] H. Widom, Asymptotic behavior of block Toeplitz matrices and determinants II, Adv. Math. 21, 1 (1976).

[34] H. Widom, On the limit of block Toeplitz determinants, Proc. Am. Math. Soc. 50, 167 (1975).

[35] M. E. Fisher and R. E. Hartwig, Toeplitz determinants, some applications, theorems and conjectures, Adv. Chem. Phys. 15, 333 (1968).

[36] E. L. Basor and K. E. Morrison, The Fisher-Hartwig conjecture and Toeplitz eigenvalues, Linear Algebra Appl. 202, 129 (1994).

[37] F. Ares, J. G. Esteve, F. Falceto, and E. Sánchez-Burillo, Excited state entanglement in homogeneous fermionic chains, J. Phys. A: Math. Theor. 47, 245301 (2014).

[38] F. Ares, J. G. Esteve, F. Falceto, and A. R. de Queiroz, On the Möbius transformation in the entanglement entropy of fermionic chains, J. Stat. Mech. (2016) 043106.

[39] F. Ares, J. G. Esteve, F. Falceto, and A. R. de Queiroz, Entanglement entropy and Möbius transformations for critical fermionic chains, J. Stat. Mech. (2017) 063104. 